\title{
Value of d-dimer test in diabetic patients as predictive marker for coronary artery disease.
}

\author{
Dr. kudair Hazbr Razzaq M.B.cH. B, DM Specialist of internal medicine (ICMS) \\ Director of diabetic center -The-Qar
}

Ali Muhain ali msc biochemistry. Diabetic center THi-Qar

\begin{abstract}
D dimer is fibrin degradation product.

Introduction:

When blood clot is degraded by fibrinolysis, natural mechanism in the body occurs when

plasminogen is activated to plasmin by fibrin.

D dimer level normally in a range around

$250 \mathrm{mg}$ per $\mathrm{ml}$. The test tends to be positive in deep venous thrombosis, pulmonary embolism, aortic dissection and post-operative state. Here in this study we try to investigate the level of $d$ dimer in the diabetic patient in comparison to non-diabetic subject. There was significant increase in the level of $\mathrm{d}$ dimer in the diabetic group. The other arm of the study is to compare the diabetic group with no clinical evidence of coronary artery disease and diabetics with documented IHD. The level was doubled and this raises the equation to initiate anti coagulation therapy early in diabetics.

$\mathrm{D}$ dimer antigen is a unique marker of fibrin degradation that is formed by sequential action of three enzymes. Thrombin, factor x111a and plasmin D dimer antigen can exist on fibrin degradation product derived from soluble fibrin before its incorporation in to fibrin gel or after the fibrin clot has been degraded by plasmin. The clinical utility of d dimer has been established in some scenario most probably for the exclusion of venous thrombo-embolsim. As well as emerging area of $\mathrm{d}$ dimer utilization as marker of coagulation activation in anther clinical setting.l. Fibrinogen is soluble plasma glycoprotein that transformed into highly self-adhesive fibrin monomer after thrombin cleavage 1 . This monomer associated with fibrinogen or fibrin to form fibrils they are held together by noncovalent forces.
\end{abstract}


Factor x111a then attach $\mathrm{d}$ dimer and insert a covalent intermolecular linkage plasmin gearedfibrin at multiple site to release fibrin .must degradation product which then expose the $\mathrm{d}$ antigen.3.Plasmin remain fluid until 25-.dimer $30 \%$ of plasma fibrinogen is cleaved by thrombin 6.Ddimer antigen remains undetectable until it is released from cross linked fibrin by the action of plasmin in the final step of $\mathrm{d}$ dimer formation plasmin formed on the fibrin surface by plasminogen activation cleave subtract fibrin at specific site .4. Fibrin degradation product are produced in wide variety of molecular weight including the terminal fibrin degradation product d $\neg$ dimer e- complex in human plasma whereas soluble high molecule weight fragment that contain the d dimer antigen are present with die and other thrombotic disorder. These fragment may be derived from soluble fibrin before it has been incorporated in to fibrin jell or alternating may be derived from high molecular weight complex reformed from an insoluble clot .5.In general the $\mathrm{d} \neg$ dimer test may be ordered to ascertain to what extent fibrin formation has been initiated or to learn whether there is any change in this process in the course of as specific therapy or disease process .6. In practice d- dimer has measurement

Been the most comprehensively validated in:

1- Exclusion of venous thrombo- embolism.
2- The diagnosis and monitoring of coagulation activation in die. The fact that only small portion of circulating fibrinogen needed to be converted to cross linked fibrin to generate a detectable $\mathrm{d}$ dimer antigen signal after plasmin digestion in plasma confers sensevity required .7 .

D dimer and deep venous thrombosis....it is used to limit the use of more expensive and invasive study. D dimer and pulmonary embolism of low clinical probability and negative $d$ dimer test effective-lyexclude pulmonary embolism. In pregnancy, in normal pregnancy d dimer levels steadily increase until the time of delivery .8. It is value in diagnosis of deep venous in pregnancy is limited 9. How ever if the $\mathrm{d}$ dimer is positive then radiographic assessment should be performed. D dimer and disseminated intravascular coagulation dimer is the most common fibrin related markers used by clinical

lab in the context.

Other uses of d dimer are diagnosis of aortic discussion; hemolysis; thrombosis cancer therapy and hema-topoietic growth factor.

\section{Method and material}

40 patient chosen randomly for this study among the attendance of the diabetic and endocrine Centre tested for d dimer and renal function test, lipid profile and blood sugar. Duration of diabetes and age of the patient was 
considered. 20 subjects tested as control group among the employee of the diabetic Centre and tested for the same parameters.

\section{Result:}

All other parameters have no effect on the level of $\mathrm{d}$ dimer and there was significant increase in diabetics. And double level in diabetic with ischemic heart disease compared to diabetics without IHD.

\section{Conclusion and recommendation:}

Is there any place for anti-coagulation in diabetics early after diagnosis? As long as the diabetics have evidence of thrombosis reflected by high levels of d dimer irrespective to age, duration, control, lipid profile?

\section{Subjects and Methods}

Samples of blood was withdrawal from 40 patients attending the endocrine and Diabetic Center in Nassyriah.And 20 normal employees in the same center as control group after having their consent. The patients were regularly interviewed and examined by physicians. The withdrawn Samples were tested in laboratories of same center. Both sampling and working were performed during the period from April 5th. 2010

to August 4th, 2011.

Sixty (60) subjects control and patient, aged (35 to 65) years were included in this study and classified as follows: -

1- Group (1): - included twenty (20) patients who were diabetic and suffering documentedischemic heart disease.

2- Group (2): - included twenty (20) patients who were diabetic and have no history of ischemie heart disease.

3- Group (3): - The control group consists of twenty (20) healthy individual. So that the normal control values for all studied parameters were obtained from them 
Data of the mentioned studied groups were shown in Table (1).

Table (1): Data of the studied groups

\begin{tabular}{|c|c|c|}
\hline Group & n & Age (Y)Mean \pm SD \\
\hline 1 & 20 & $54.4 \pm 6.9$ \\
\hline 2 & 20 & $49.6 \pm 6.6$ \\
\hline 3 & 20 & $41.8 \pm 7.4$ \\
\hline
\end{tabular}

$\mathrm{N}$ : Number of subject.

SD: Standard deviation.

\section{Y: Years.}

The sample of blood $(5 \mathrm{ml})$ was withdrawn from randomly selected subject by vein puncture and transferred into empty disposable tube's centrifuge to separate it, after the clotting, in the centrifuge at 3000 rotors per minute (rpm) for 10 min the.
Serum samples were separated from the cells promptly stored on ice at $\left(-20^{\circ} \mathrm{C}\right)$ to use it after time to measure the biochemical parameters. D-Dimer (DD2) is determined using the vidas instruments Vida's D-Dimer exclusion is an automated quantitative test for use on the Vida's instruments for immunoenzymatic determination for fibrin degradation product (FbDP) in human plasma (sodium citrate) using the Enzyme Linked Fluorescent Assay technique (ELFA) (1'2). 
Thi-Qar Medical Journal (TQMJ): Vol.(11), No.(1), 2016

utjmed@utq.edu.iq
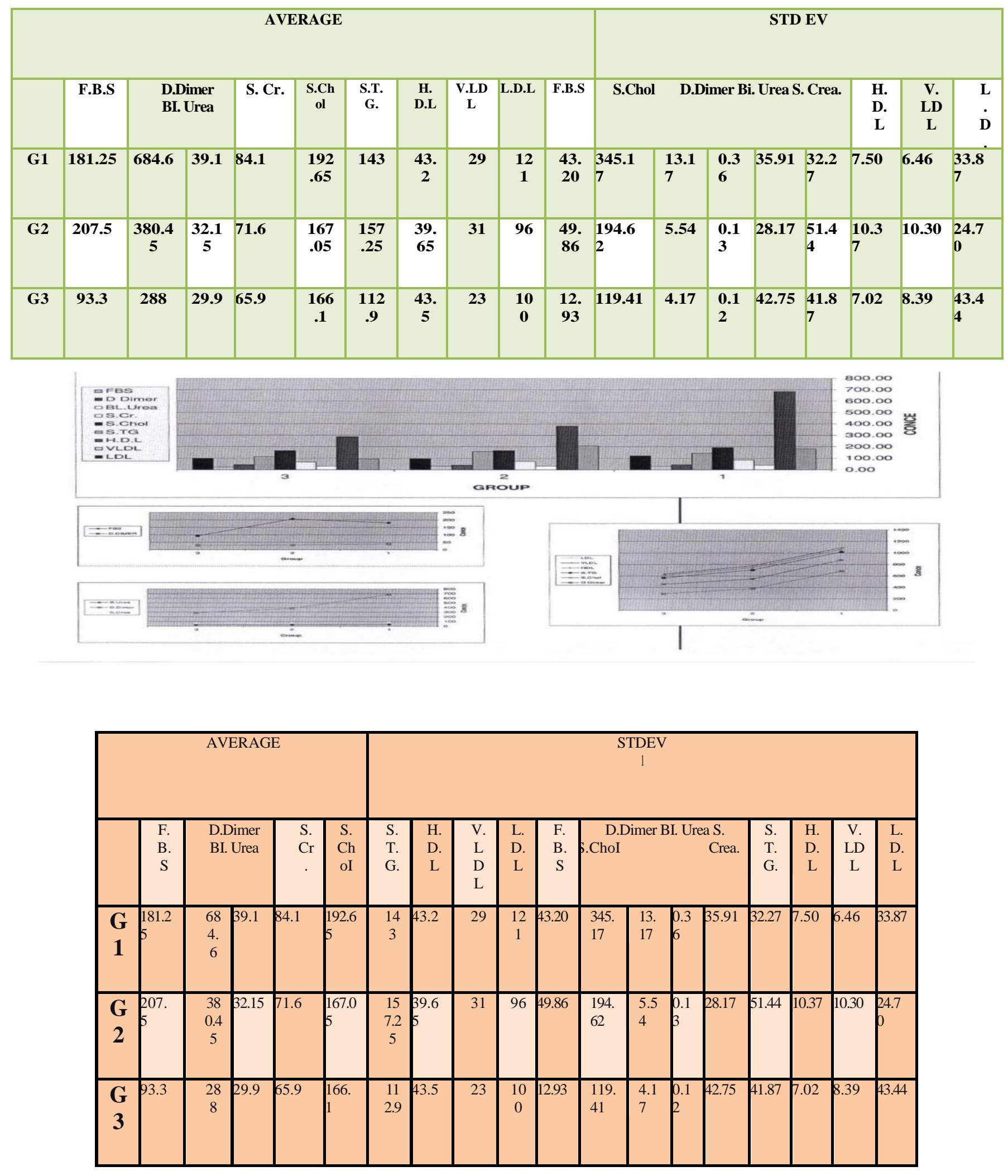


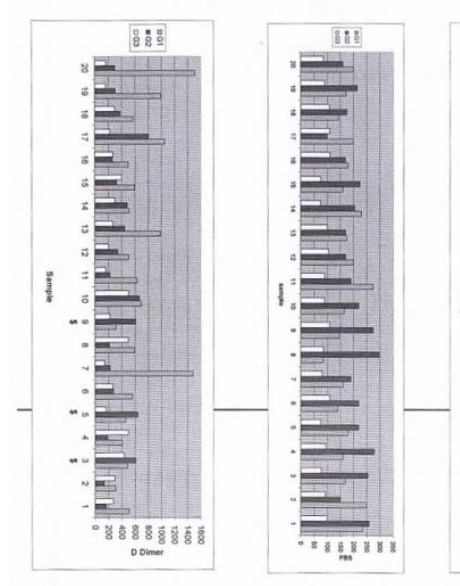

The d-dimer occure in normal range of S00 ng-ml the d-dimer level testing to check for pulmonary embolism. negative $\mathrm{d}$ - dimer will almost role out the possibility of PE.

D-dimer test will reduce the probability of thromboembolic disease is dependent of the test. d-dimer test claims to have $93-95 \%$ sensevity and $50 \%$ specifcity for diagnosis thrombotic disease. False positive test may result from liver disease inflammatory process malignant tuomer physical trauma.

Here in this study we try to investigate the level of d- dimer in diabetic patient in comparsion to non-diabetic subject and diabetic subject with documented ischemic heart disease.

\section{Notes on the results obtained from the study}

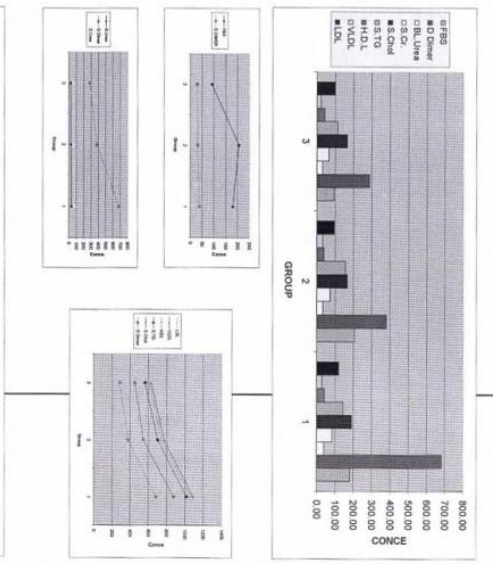

Results and Discussion

1- D-dimer level increament doesn't relate to period of diabetes mellitus.

2- D-dimer not related to age.

3- D-dimer not related to control of blood suger.few result may have revers proportion to blood suger control.

4- D-dimer not related to lipid profile.

5- There is signifant increase of d- dimer in diabetic patient.

6- duoble increase in diabetic patient with coronary artery disease.

We need to have more studies to support this study.

\section{Recommendations}

Quation raised to recommend anticoagulant theray in diabetics once diagnosed if $\mathrm{d}$ dimer is signifacly raised as this fact reflect an evidenceof occult thrombosis. 


\section{References:}

1- Blomback B; hessl B; Hogged Tow step fibrinogen fibrin in blood coagulation.

2- Fowler WE; Grickson HP'Hantgan RR; Mc. Donagh J; Hermans J Cross linked fibrinogen ddimers demonstrate features of molecular packing in fibrin fibers.

3- Green berg CS; Miralgia CC; Rickies FR; shuman MA Cleavage of blood coagulation factor x111 and fibrinogen by thrombin in vitro dotting.

4- Medved L; nieuwenhuizen W Molecular mechanism of intiation of fibrinolysis by fibrin.

5- Marden VT; Zareba W; Horan JT; Moss AJ; Kanonse J JAutomated latex agglutination and elisa testing yield equivalent d-dimer result in patient with recent myocardial infarction.

6- Lowe GD Fibrin d-dimer and cardiovascular disease.

7- Ems MT; Bunce IH; Bundesen PG Measurement of cross linked degredation product.

8- Cichingers d-dimer testing in pregnancy.

9- Chan W.S; Chunilal; S; Lee A Red blood cell agglutination d-dimer to exclude DVT in pregnancy 